\title{
EDITORIAL
}

\section{Conflicts of interest in intensive care medicine}

\author{
Dustin C. Krutsinger ${ }^{1,2,3^{*}}$, Scott D. Halpern ${ }^{1,2,3,4}$ and Erin S. DeMartino 5,6
}

(0) 2018 Springer-Verlag GmbH Germany, part of Springer Nature and ESICM

Conflicts of interest in medicine are pervasive and are not isolated to certain practice settings or specialties. The Institute of Medicine defines a conflict of interest as "a set of circumstances that creates a risk that professional judgement or actions regarding a primary interest will be unduly influenced by a secondary interest" [1]. Medical professionals have a duty to minimize and disclose conflicts of interest in order to preserve the trust of patients and the public. In this editorial we focus on financial personal conflicts, non-financial personal conflicts, and conflicts that arise naturally in the role of ICU clinician, highlighting facets shared across medicine and challenges unique to the practice of intensive care medicine.

\section{Financial personal conflicts}

ICU providers may have financial ties to investigational devices, proprietary assays or medications, or collect speakers' fees. Yet advances in intensive care medicine have not traditionally been industry-driven to the same extent as in other fields, such as oncology or cardiology. With several notable exceptions such as drotrecogin alfa [2] and dexmedetomidine [3], most contemporary trials in critical care involve generic medications with other established uses (steroids, paralytics or resuscitative fluids) or non-pharmacologic interventions (prone positioning, ventilation strategies or early vs. late renal replacement therapy). To the extent any financial personal conflicts exist, intensivists should comply with reporting mechanisms such as the Centers for Medicare and Medicaid Service's Open Payments system [4]. The reimbursement structure in the United States may impact treatment decisions in ICUs, though citing

\footnotetext{
*Correspondence: Dustin.Krutsinger@uphs.upenn.edu

${ }^{1}$ Department of Medicine, Perelman School of Medicine, University of Pennsylvania, Philadelphia, PA, USA

Full author information is available at the end of the article
}

fee-for-service as a primary determinant of decision making over-simplifies complex practice patterns [5].

\section{Non-financial personal conflicts}

Although financial conflicts of interest deservedly receive attention from professional societies and policymakers, non-financial personal conflicts may be even more prevalent in ICUs. Advancement of scientific knowledge, practice innovation and improvements in patient outcomes drive the majority of ICU investigators. However, secondary motivations such as career advancement and job security are predicated on research productivity, as measured by prolific publication and securing extramural funding. Pressure to enroll patients in clinical trials may lead to inadequate disclosure of risks, exaggeration of potential benefits of study participation, enrollment of subjects who do not meet eligibility criteria, failure to disclose adverse events to oversight committees, data manipulation and failure to terminate trials when indicated [6, 7]. A review of the incidents of research misconduct reported on the US Department of Health and Human Services Office of Research Integrity [8] suggested that falsified data and other misconduct are rarely traced to direct financial conflicts of interest, but are more likely to stem from academic pressures such as securing extramural funding, publishing manuscripts and advancing in academic rank [9]. Moreover, institutional pressure to establish and maintain research funding or meet enrollment rates set by clinical trial networks may influence patient recruitment practices [6].

The 2004 American Thoracic Society guidelines on the ethical conduct of clinical research recognize that "clinicians who wish to enroll their own patients in studies have an inherent conflict of interest in that the desire to collect generalizable knowledge can conflict with individual patient care". The guidelines recommend that a third

\section{Springer}


party obtain consent to maintain a separation between clinical and research practices [10].

\section{Role conflicts}

In addition to conflicts related to financial and professional advancement, intensivists face several role conflicts in the course of caring for critically ill patients.

Intensivists often serve as gatekeepers to the ICU. This role reveals a fundamental conflict between serving as patient advocate for patients who already occupy ICU beds, and serving as steward of ICU resources when considering other individuals who could potentially benefit from ICU admission. It is commonly held that ICU triage should not follow a first-come, first-served protocol, and should instead prioritize patients with the greatest likelihood of benefit [11]. Extension of that principle could involve transferring patients who have not improved after a time-limited trial of intensive care in order to accommodate patients for whom intensive care might confer a greater survival benefit. However, most intensivists report prioritizing patients already hospitalized in an ICU who continue to warrant ICU care over the acceptance of new patients [11]. This apparent contradiction reflects the conflict intensivists face when weighing the interests of their individual patients against the ethical obligations of allocating scare resources.

At times, the physician's role as caretaker and educator conflict. As in other fields, training tomorrow's physicians requires trade-offs between supervision and autonomy [12]. These considerations are highlighted when trainees must determine treatment plans for unstable patients or must become proficient at performing invasive procedures. As a medical profession, we rarely acknowledge that while medical education benefits society, it exposes individual patients to risks with little prospect of benefit. For the ethical maintenance of this system, proper oversight for trainees is essential.

Finally, intensivists care for patients who may eventually become eligible for organ donation. Indeed, some physicians may hold a dual appointment in a transplant center. The organ procurement process has long been identified as a system at risk for conflicts of interest that could jeopardize public trust. These risks have been mitigated by separating the treating physicians from the organ donation decision and procurement processes.

Physicians with financial and non-financial personal conflicts should communicate, mitigate and divest in order to minimize impact on care decisions for their patients. A nuanced examination of the practice of intensive care medicine also exposes a number of non-financial role conflicts, including those between the role of advocating for patients currently under one's care and stewarding scarce resources responsibly to promote public health, and, in academic settings, between the roles of physician, educator and investigator. Acknowledging these inherent tensions is a prerequisite for intensivists to responsibly balance the interests of individual patients against responsibilities to triage, educate and advance medical knowledge through research. However, ultimately, the creation and maintenance of a culture of ethical practice within each ICU is necessary to minimize the impact of these conflicts on patients.

\section{Author details \\ ${ }^{1}$ Department of Medicine, Perelman School of Medicine, University of Penn- sylvania, Philadelphia, PA, USA. ${ }^{2}$ Palliative and Advanced IIIness Research Center, University of Pennsylvania, Philadelphia, PA, USA. ${ }^{3}$ Center for Health Incentives and Behavioral Economics at the Leonard Davis Institute of Health Economics, Perelman School of Medicine, University of Pennsylvania, Phila- delphia, PA, USA. ${ }^{4}$ Department of Medical Ethics and Health Policy, Perelman School of Medicine, University of Pennsylvania, Philadelphia, PA, USA. ${ }^{5}$ Divi- sion of Pulmonary and Critical Care Medicine, Mayo Clinic, Rochester, MN, USA. ${ }^{6}$ Biomedical Ethics Research Program, Mayo Clinic Rochester, Rochester, MN, USA.}

Received: 10 July 2018 Accepted: 24 July 2018

Published online: 2 August 2018

\section{References}

1. Steinbrook R (2009) Controlling conflict of interest-proposals from the Institute of Medicine. N Engl J Med 360:2160-2163

2. Ranieri VM, Thompson BT, Barie PS et al (2012) Drotrecogin alfa (activated) in adults with septic shock. N Engl J Med 366:2055-2064

3. Riker RR, Shehabi Y, Bokesch PM et al (2009) Dexmedetomidine vs midazolam for sedation of critically ill patients: a randomized trial. JAMA 301:489-499

4. Centers for Medicare and Medicaid Services. Open Payments. https:// www.cms.gov/openpayments. Accessed 5 June 2018

5. Rotenstein $L$, Jena $A B(2018)$ It's time to rethink the anatomy of physician behavior. Health Affairs Blog: Health Affairs. https://www.healthaffairs. org/doi/10.1377/hblog20180618.721948/full/. Accessed 29 June 2018

6. Levinsky NG (2002) Nonfinancial conflicts of interest in research. N Engl J Med 347:759-761

7. Rosenberg AR (2017) "Get the consent"-nonfinancial conflict of interest in academic clinical research. J Clin Oncol 35:11-13

8. U.S. Department of Health and Human Services Office of Research Integrity Case Summaries. https://ori.hhs.gov/content/case_summary. Accessed 29 June 2018

9. Saver RS (2012) Is it really all about the money? Reconsidering nonfinancial interests in medical research. J Law Med Ethics 40:467-481

10. Luce JM, Cook DJ, Martin TR et al (2004) The ethical conduct of clinical research involving critically ill patients in the United States and Canada: principles and recommendations. Am J Respir Crit Care Med 170:1375-1384

11. Sprung CL, Danis M, lapichino G et al (2013) Triage of intensive care patients: identifying agreement and controversy. Intensive Care Med 39:1916-1924

12. Chiong W (2007) Justifying patient risks associated with medical education. JAMA 298:1046-1048 